\title{
Prevalencia y factores de riesgo de comportamientos relacionados con suicidio en el Estudio Nacional de Salud Mental, Colombia 2003 (ENSM)
}

\author{
José Posada-Villa, MD1, Marcela Rodríguez, MD¹, Patricia Duque, MSc¹, \\ Alexandra Garzón, MSc ${ }^{1}$ \\ ${ }^{1}$ Grupo de Investigaciones Nuevas perspectivas en Salud Mental; Facultad de Ciencias Sociales; \\ Universidad Colegio Mayor de Cundinamarca \\ Correspondencia: hmontoyap@yahoo.com \\ Recibido: 14-05-2007 / Aceptado: 6-06-2007
}

\begin{abstract}
Resumen
El suicidio es un problema importante de salud pública en Colombia. Sin embargo, poco es conocido acerca de la prevalencia y factores de riesgo de los principales comportamientos relacionados con este. El presente artículo intenta mostrar la prevalencia y factores de riesgo en el suicidio, a partir de los resultados de la encuesta nacional de salud mental de Colombia. Los parámetros evaluados fueron la ideación, los planes y los intentos de suicidio en una encuesta de hogares, realizada cara a cara, con una muestra representativa a nivel nacional de 4426 adultos, que hace parte de la Encuesta Mundial de Salud Mental de la Organización Mundial de la Salud. La prevalencia de ideación, de planes e intentos de suicidio son $12.4 \%$ (0.7), 4.1\% (0.4) y 4.7\% (0.4) respectivamente. El mayor riesgo tanto de ideación como de intento de suicidio entre aquellos con ideación es en el grupo entre 18-29 años. En Colombia como en el mundo, el suicidio es una entidad de cuidado, con una prevalencia importante y sobre la cual se deben hacer esfuerzos para disminuir los números arrojados por este estudio. Los esfuerzos para la prevención del suicidio son desafortunadamente y con urgencia necesarios en Colombia. Por lo tanto, futuras investigaciones deben definir los determinantes de los comportamientos relacionados con el suicidio.
\end{abstract}

Palabras claves: ansiedad, ideación suicida, salud mental, salud pública, suicidio, trastorno mental

\footnotetext{
Abstract

Suicide is an important public health issue in Colombia. Nevertheless, little is known about the prevalence, risk factors, and the main behaviors related to it. The present article tries to show to the prevalence and suicide risk
} 
factors based on the results of the national survey of mental health of Colombia. The evaluated parameters were the thought, plans, and suicide attempts in a survey of homes, made face to face, with a representative sample at national level of 4426 adults, which makes part is of the World-wide Survey of Mental Health of the World Health Organization. The prevalence of thought, plans, and attempts of suicide is $12,4 \%(0.7) ; 4,1 \%(0.4)$; and $4,7 \%(0.4)$ respectively. The highest risk of both suicide ideation and attempt is in the 18-29 age group. In Colombia, and in the world, suicide is an issue to care about because it has an important prevalence. In Colombia, suicide prevention efforts are unfortunately necessary to diminish the numbers shown by this study. Therefore, future investigations must define the determinants of the behaviors related to suicide.

Key words: Anxiety, mental disorders, mental health, public health, suicidal thought, suicide.

\section{Introducción}

En Colombia, según datos del Instituto Nacional de Medicina Legal y Ciencias Forenses, la tasa de suicidios durante 2003 fue de 4.4 (1). Ante esta situación, los estudios realizados en Colombia han revelado la importancia de programas de prevención de este fenómeno, como se ha puntualizado en el Plan Nacional de Desarrollo que actualmente está para la firma del Presidente de la República, en la Ley 1122 de 2007 que reforma el Sistema de Seguridad Social en salud y el Plan Nacional de Salud Pública 2007-2010 de agosto de este año.

A pesar de estas circunstancias, los datos básicos sobre la prevalencia y los factores de riesgo para sus precursores inmediatos -ideación, planes e intentos de suicidio- son escasos en población general a nivel nacional. La información sobre comportamientos relacionados con los suicidios recolectados en Colombia, podrían iluminar de gran manera la investigación, las políticas y los esfuerzos dirigidos al entendimiento y la prevención del suicidio en todo el país.

El propósito de este estudio fue estimar la prevalencia de los comportamientos relacionados con el suicidio (ideación, plan e intento de suicidio) y examinar los potenciales factores de riesgo para esos comportamientos, utilizando datos del Estudio Nacional de Salud Mental, Colombia 2003, realizado como parte de la World Mental Health (WMH) Survey Initiative de la Organización Mundial de la Salud (OMS) (2).

\section{Métodos}

\section{Muestra de respondientes}

El Estudio nacional de salud mental, Colombia 2003 (ENSM) se basa en un diseño probabilístico, multietápico y estratificado para cinco regiones del país, cuya población blanco fue la población no institucionalizada, que tiene un hogar fijo, de 18 a 65 años de edad y que vive en áreas urbanas. La tasa de respuesta ponderada fue de $87,7 \%$. Los datos fueron recolectados en 60 municipios representativos del país, utilizando las definiciones aceptadas de comportamientos relacionados con el suicidio (3) y con métodos de medición altamente consistentes. El tamaño total de la muestra fue de 4426, y la tasa de respuesta fue de $88.7 \%$ (4)

\section{Procedimiento}

Todos los entrevistados completaron la Parte I de la encuesta que contenía una evaluación de los principales diagnósticos psiquiátricos, incluyendo la evaluación de comportamientos relacionados con el suicidio. Todas las personas encuestados de la Parte I, que reunían criterios para cualquier diagnóstico y una submuestra de aproximadamente $25 \%$ de los que no reunían criterios diagnósticos, se les realizó la Parte II de la encuesta, que evaluó los correlatos potenciales y diagnósticos de interés secundario. Estos datos fueron ponderados, ajustados a las probabilidades de diferenciales de selección entre hogares y para adecuar las muestras a la distribución sociodemográfica de la población. 
Los procedimientos estandarizados de entrenamiento de los encuestadores, los protocolos de traducción de la OMS para todos los materiales del estudio, los procedimientos de control de calidad para los encuestadores y la precisión de los datos son descritos con mayor detalle en otra publicación (5). Se obtuvo consentimiento informado y la manera de proteger los derechos humanos de los sujetos fue aprobada y monitorizada en su cumplimiento por el comité asesor del estudio bajo la coordinación del Ministerio de la Protección Social

\section{Medidas de comportamientos relacionados con suicidio}

Los comportamientos relacionados con suicidio fueron evaluados utilizando la versión 15 de la Composite International Diagnostic Interview (CIDI) de la OMS (6). La WHO/CIDI contiene un modulo que evalúa ideación suicida (“¿Alguna vez ha pensado seriamente en suicidarse?”), planes suicidas (“¿Alguna vez ha hecho un plan para suicidarse?"), e intentos de suicidio (“¿Alguna vez ha intentado suicidarse?”). Las entrevistas evaluaban la presencia durante la vida y la edad de aparición de cada resultado.

\section{Factores de riesgo para comportamientos relacionados con suicidio}

La encuesta también examinó tres grupos de factores de riesgo para comportamientos relacionados con el suicidio: factores sociodemográficos, características de los comportamientos relacionados con el suicidio y trastornos mentales previos según la DSM-IV. Los factores sociodemográficos incluyeron: género, edad/ cohorte, educación, historia de empleo e historia marital. Las características de los comportamientos examinados relacionados con suicidio incluyeron: edad de aparición de la ideación, tiempo desde la aparición de la idea, presencia de plan suicida y tiempo desde la aparición del plan. Los trastornos mentales de los encuestados fueron evaluados utilizando el WHO CIDI (3). La evaluación incluyó trastornos del estado de ánimo, de ansiedad, del control de impulsos y por uso de sustancias. Fueron utilizadas reglas de exclusión de organicidad y de jerarquía al hacer todos los diagnósticos de los entrevistados. Estudios previos utilizando reevaluaciones clínicas encontraron que el diagnóstico con la CIDI tiene generalmente buena concordancia con diagnósticos basados en la entrevista clínica estructurada del DSMIV en una submuestra probabilística de entrevistados de la encuesta realizada en los Estados Unidos dentro de la Encuesta Mundial de Salud Mental(7).

\section{Análisis estadístico}

Fue utilizada una tabulación cruzada para estimar la prevalencia de vida de ideación, planes e intentos de suicidio. También se utilizaron análisis de regresión logística multivariada (8) para estimar las asociaciones de correlatos sociodemográficos y diagnósticos retrospectivamente evaluados de cada resultado. Los coeficientes de regresión logística e intervalos de confianza (IC) de $95 \%$ fueron convertidos en odd-ratios (OR) para facilitar la interpretación. Las variables continuas fueron divididas en categorías para minimizar efectos de valores extremos. Los errores estándar (EE) y los test de significancia fueron calculados utilizando el método de series de Taylor (9) y el software SUDAAN (10) para ajustar por efectos de diseño. La significancia multivariada fue evaluada utilizando el test de Wald $\mathrm{X}^{2}$.

\section{Resultados}

\section{Prevalencia}

La prevalencia de vida estimada (ES) de ideación, plan e intento de suicidio en toda la muestra fue de $12.4 \%$ (0.7), $4.1 \%(0.4)$ y $4.7 \%(0.4)$, respectivamente, Tabla 1. Entre aquellos con ideación suicida, la probabilidad condicional de hacer alguna vez un plan suicida fue $18.8 \%$ (2.6) y de hacer alguna vez un intento de suicidio fue $17.0 \%$ (2.5). La probabilidad de intento entre aquellos con ideación con un plan fue $52.1 \%$ (7.0), pero solo el $8.8 \%$ (2.1) entre aquellos sin un plan. 
Tabla 1. Prevalencia de vida de comportamientos relacionados con suicidio: ENSM, Colombia 2003

\begin{tabular}{|c|c|c|c|c|c|c|c|c|c|c|c|c|c|c|c|c|c|c|c|c|c|}
\hline & \multicolumn{3}{|c|}{ Ideación } & \multicolumn{2}{|l|}{ Plan } & \multicolumn{3}{|c|}{ Intento } & \multicolumn{3}{|c|}{$\begin{array}{l}\text { Plan entre } \\
\text { ideadores }(n=587)\end{array}$} & \multicolumn{3}{|c|}{$\begin{array}{l}\text { Intento entre ideadores } \\
(n=587)\end{array}$} & \multicolumn{3}{|c|}{$\begin{array}{l}\text { Intento entre } \\
\text { ideadores sin plan } \\
\text { durante la vida } \\
(n=383)\end{array}$} & \multicolumn{4}{|c|}{$\begin{array}{l}\text { Intento entre ideador } \\
\text { es con plan durante la vida } \\
(n=204)\end{array}$} \\
\hline & $\%$ & SE & $n$ & $n$ & $\%$ & SE & $n$ & $\%$ & SE & $n$ & $\%$ & SE & $n$ & $\%$ & SE & & $n$ & $\%$ & SE & & $n$ \\
\hline Hombres & 11.9 & 0.9 & 201 & 3.6 & 0.5 & 70 & 4.7 & 0.7 & 73 & 30.5 & 3.8 & 70 & 39.4 & 4.1 & 73 & 27.7 & 5.4 & 28 & 66.1 & 5.9 & 45 \\
\hline Mujeres & 12.7 & 1.0 & 386 & 4.5 & 0.6 & 134 & 4.6 & 0.5 & 151 & 35.3 & 3.3 & 134 & 36.6 & 2.8 & 151 & 18.5 & 3.4 & 49 & 69.8 & 6.0 & 102 \\
\hline $\begin{array}{l}\text { Muestra } \\
\text { total }\end{array}$ & 12.4 & 0.7 & 587 & 4.1 & 0.4 & 204 & 4.7 & 0.4 & 224 & 33.2 & 2.6 & 204 & 37.8 & 2.6 & 224 & 22.7 & 3.2 & 77 & 68.3 & 3.5 & 147 \\
\hline
\end{tabular}

\section{Factores socio-demográficos}

La ideación y el intento de suicidio son significativamente más comunes en las cohortes de edad más recientes que en las más tempranas $(\mathrm{OR}=5.5)$, Tabla 2. En la edad/ cohorte, el mayor riesgo tanto para ideación como intento de suicidio es en la de 35-49 años $(3.5,68.1)$, seguido por 18-34 años $(3.1,37.7)$, 50-64 años $(2.2,32.6)$. Las relaciones entre factores de riesgo sociodemográficos y comportamientos relacionados con suicidio están atenuadas cuando predicen planes e intentos de suicidio entre aquellos con ideación, sugiriendo que las asociaciones de estos factores sociodemográficos con planes e intentos de suicidio son en gran medida por ideación suicida, Tabla 2.

\section{Características de comportamientos relacionados con suicidio como factores de riesgo}

Para examinar la relación entre edad de aparición y riesgo de transición de ideaciones a planes y a intentos, aquellos con ideación suicida fueron clasificados en terciles basados en la edad de aparición esta. Los análisis revelaron que una edad de inicio temprana está asociada con un gran riesgo de plan e intento de suicido entre aquellos con ideación suicida, aunque esta asociación no es estadísticamente significativa. La transición de ideación suicida a primera aparición de plan o intento suicida es mayor entre el primer año de aparición de la ideación suicida $(\mathrm{OR}=170.1-390.2)$, y disminuye sustancialmente después ( $\mathrm{OR}=1.4-3.2)$. Tener un plan suicida está asociado con un mayor riesgo de alguna vez hacer intento de suicidio entre aquellos que tienen ideas de suicidio $(\mathrm{OR}=32.7)$, aunque el "odds" para hacer un intento entre el primer año de la aparición de ideación suicida es también muy alto para aquellos sin un plan (por ejemplo, intentos de suicidio impulsivo; $(\mathrm{OR}=122609.8)$. Entre aquellos que intentan con un plan, la transición a intento de suicidio es mayor dentro del primer año de tener un plan suicida $(\mathrm{OR}=5157.8)$.

\section{Trastornos mentales como factores de riesgo}

La presencia de un trastorno mental previo está asociado con un significativo incremento de riesgo de la subsiguiente primera aparición de ideación, plan e intento de suicidio, aún después de controlar los factores y características sociodemográficas de los comportamientos relacionados con suicidio, Tabla 3. El riesgo de comportamientos relacionados con suicidio es mayor entre trastornos del estado de ánimo $(\mathrm{OR}=5.5$, $6.9,19.4)$, seguido por trastorno por consumo de sustancias $(3.9,5.2,7.7)$ y trastornos de ansiedad (3.3, $5.9,5.1)$. Asociaciones entre trastornos mentales y comportamientos relacionados con suicidio son atenuados cuando predicen planes e intentos entre personas con ideación, con OR decreciendo a 0.9-8.5 a través de todas las categorías, sugiriendo que los efectos de los trastornos mentales son en su mayor parte sobre la ideación. Entre 
personas con ideación, el riesgo de hacer un intento es mayor para aquellos con trastornos del estado de ánimo. Los resultados también muestran una fuerte relación dosis-respuesta entre el número de trastornos mentales presentes y el riesgo de cada resultado relacionado con suicidio, Tabla 3.

\section{Discusión}

Los hallazgos deben ser interpretados en el contexto de varias limitaciones importantes. Primero, los datos están basados en un auto reporte retrospectivo de la ocurrencia y momento de conductas relacionadas con el suicidio y trastornos mentales, y por lo tanto puede ser sujeto de sub-reporte y recuerdos sesgados. No recolectamos información de personas en el entorno del entrevistado para validar los reportes de estos. Sin embargo, se han publicado varias revisiones sistemáticas que han demostrado que los adultos pueden recordar experiencias pasadas con suficiente exactitud para dar información de valor $(11,12)$. Tales datos son especialmente útiles, por supuesto, cuando no hay disponibles, (7) como en el caso presente.

Otra limitación digna de tenerse en cuenta es que varios trastornos mentales importantes no fueron evaluados en la Encuesta Mundial de Salud Mental (WMH surveys) porque estas podrían ser difíciles de entrevistar debido al estigma y porque previos estudios de validación demostraron que ellas son sobre-estimadas en entrevistas administradas por legos como la CIDI, (13) como en el caso de los trastornos psicóticos. Esto es desafortunado dado que estudios previos sugieren que la esquizofrenia y los comportamientos relacionados con suicidio participan de patrones de prevalencia únicos. Una limitación relacionada fue la de comportamientos de auto-lesión no suicidas, tales como gestos suicidas (14) y auto-mutilación (15) no fueron considerados en este estudio.

No obstante estas limitaciones, los resultados dan una información invaluable y previamente no disponible sobre las tasas de comportamientos relacionados con suicidios no letales en Colombia. Es de anotarse que la cohorte de 35-49 años de edad y después del primer empleo son factores de riesgo en Colombia, son diferentes cuando se les compara con reportes previos en otros países $(16,17)$ en los que el genero femenino, ser joven y tener desventaja social, han sido reportados como factores de riesgo para comportamientos relacionados con el suicido. La edad media es un factor de riesgo y es consistente con las tendencias recientes del altísimo incremento de tasas de suicidio entre los 30 y 40 años de edad. De acuerdo con las estadísticas del Instituto Nacional de Medicina Legal y Ciencias Forenses, por ejemplo, el más importante incremento de la tasa de suicidio se da entre 30 años (tasa de incremento, 8.6\%), seguido por 50 años (7.3\%) y 40años (3.7\%) en el 2002 y entre 30 años (17.0), 40años (12.6\%) y 20años (11.1.) en el 2003.

El riesgo de planes e intentos de suicidio también fueron altos cuando la ideación suicida tuvo una temprana edad de aparición y dentro del primer año de ideación suicida. Un gran número de las transiciones de ideación a plan y a intento ocurren dentro del primer año de aparición de la ideación. Muy pocos estudios previos han examinado la probabilidad y velocidad de transición de la ideación al plan y al intento. Esta información puede ser especialmente útil para los proveedores de atención en salud que trabajan con individuos potencialmente suicidas. Otro punto importante de anotar, tanto para propósitos clínicos como científicos, es que la fuerte relación observada entre trastornos mentales y planes suicidas e intentos, disminuyen de manera significativa cuando se controlan por ideación. Esto sugiere que factores más allá de la mera presencia de la enfermedad mental explican la transición de ideación a planes e intentos. La severidad de los trastornos mentales puede ser crítica en la contabilidad para estas transiciones junto con otros factores que son independientes de los trastornos mentales.

Ha sido sugerido que más del $90 \%$ de aquellos que mueren por suicidio tienen un trastorno mental diagnosticable. Nuestros hallazgos indican que los trastornos mentales son predictivos de comportamientos 


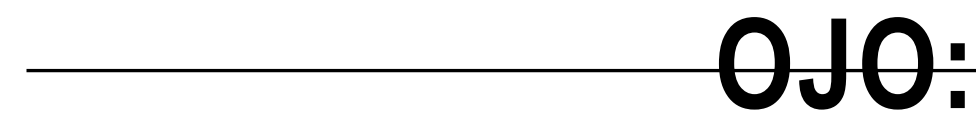

PARA SUSTITUIR CON EL IN-SERTO ADJUNTO EN PDF

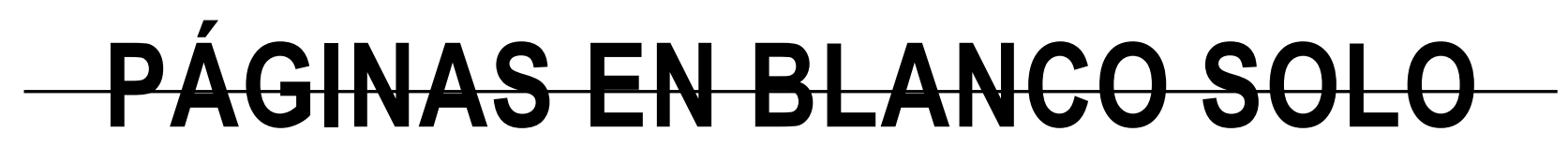
PARA REFERENCIA EN EL MONTAJE 
OJO:

PARA SUSTITUIR CON EL IN-SERTO ADJUNTO EN PDF

PÁGINAS EN BLANCO SOLO

PARA REFERENCIA EN EL MONTAJE 
relacionados con los suicidios examinados aquí y que la comorbilidad es un factor predictor importante en nuestro medio. Los trastornos del estado de ánimo fueron más predictivos de comportamientos relacionados con el suicidio como se reporta en estudios previos.

Estos hallazgos tienen considerables implicaciones tanto científicas como prácticas. Desde la perspectiva científica, dan información de valor acerca de la ocurrencia de comportamientos relacionados con el suicidio y son consistentes con muchos hallazgos previos. Es importante realizar investigaciones que incluyan el examen de determinantes de la prevalencia y la evaluación de modelos más complejos de factores de riesgo y protectores que toman en cuenta tanto aspectos comunes como específicos para Colombia. Desde una perspectiva práctica, nuestros datos dan una información importante para los investigadores del país para examinar la efectividad de los programas de intervención comunitarios para prevenir el suicidio y los intentos de suicidio y sobre todo el seguimiento a los casos que llegan a los servicios de urgencias.

\section{Agradecimientos}

El Estudio Nacional de Salud Mental, Colombia 2003 fue llevado a cabo conjuntamente con la World Health Organization World Mental Health (WMH) Survey Initiative (http://www.hcp.med.harvard.edu/wmh/). Agradecemos a los miembros del equipo coordinador de la WMH por su apoyo con la instrumentación y su asesoría en el trabajo de campo. Estas actividades fueron apoyadas por el United States National Institute of Mental Health (R01MH070884), the John D. and Catherine T. MacArthur Foundation, la Pfizer Foundation, the US Public Health Service (R13MH066849, R01-MH069864, and R01 DA016558), el Fogarty International Center (FIRCA R01-TW006481), la Pan American Health Organization, Eli Lilly and Company, Ortho-McNeil Pharmaceutical, Inc., GlaxoSmithKline, and Bristol-Myers Squibb. El estudio en Colombia fue realizado con recursos del Ministerio de la Protección Social. Queremos agradecer a la Fundación FES Social por su participación en el trabajo de campo. También agradecemos a los miembros de grupo de trabajo sobre suicidio de la WMH.

\section{Referencias}

1. Instituto Nacional de Medicina Legal y Ciencias Forenses Forensis 2003. Bogotá: Imprenta Nacional.

2. WHO World Mental Health Consortium. Prevalence severity, and unmet need for treatment of mental disorders in the World Health Organization World Mental Health Surveys. JAMA 2004;291:2581-2590.

3. Kessler R, Ustun T. The World Mental Health (WMH) Survey Initiative Version of the World Health Organization (WHO) Composite International Diagnostic Interview (CIDI). Int J Methods Psychiatr Res. 2004;13:93-121.

4. Posada-Villa J, Aguilar-Gaxiola, S., Magaña, C, Gómez L. Prevalencia de trastornos mentales y uso de servicios: Resultados preliminares del Estudio Nacional de Salud Mental Colombia, 2003 Rev Colomb Psiquiatr. 2004;33:241-26.

5. Kessler R, Abelson J, Demler O, Escobar J. Gibbon M, Guyer M Howes M, Jin R, Vega W, Walters E, Wang P, Zaslavsky A, Zheng H. Clinical Calibration of DSM-IV Diagnoses in the World Mental Health (WMH) Version of the World Health Organization (WHO) Composite International Diagnostic Interview (CIDI). Int J Methods Psychiatr Res 2004;13:122-139.

6. Posada-Villa J, Trevisi C. Prevalencia, severidad y necesidades no satisfechas del tratamiento de los trastornos de ansiedad, relacionados con sustancias, del estado de ánimo y del control de los impulsos en adultos según el Estudio Nacional de Salud Mental, Colombia 2003. MedUNAB, 2004;7:65-72.

7. Kessler R, Berglund P, Demler O, Jin R, Walters E. Lifetime prevalence and age-of-onset distributions of DSM-IV disorders in the National Comorbidity Survey Replication (NCS-R). Arch Gen Psychiatry. 2005:62:593-602.

8. Hosmer DW, Lemeshow S. Applied Logistic Regression. New York: Wiley Sons, 1989.

9. Wolter K. Introduction to Variance Estimation. New York, Springer-Verlag, 1985

10. SUDAAN: Professional Software for Survey Data Analysis (program). 8.01 version. Research Triangle Park, NC: Research Triangle Institute, 2002.

11. Brewin C, Andrews B, Gotlib I. Psychopathology and early experience: a reappraisal of retrospective reports. Psychol Bull. 1993;113:82-98.

12. Hardt J, Rutter M. Validity of adult retrospective reports of adverse childhood experiences: review of the evidence. J Child Psychol Psychiatry. 2004;45:260-273.

13. Kendler K, Gallagher T, Abelson J, Kessler R. Lifetime prevalence, demographic risk factors, and diagnostic validity of nonaffective psychosis as assessed in a US community sample. The National Comorbidity Survey. Arch Gen Psychiatry. 1996;53:1022-1031.

14. Nock M, Kessler R. Prevalence of and risk factors for suicide attempts versus suicide gestures: Analysis of the National Comorbidity Survey. J Abnorm Psychol. 2006;115:616-623.

15. Nock M, Prinstein M. Contextual features and behavioral functions of self-mutilation among adolescents J Abnorm Psychol. 2005;114:140-146.

16. Kessler R, Borges G, Walters E. Prevalence of and risk factors for lifetime suicide attempts in the National Comorbidity Survey. Arch Gen Psychiatry. 1999;56:617-626.

17. Moscicki E. Epidemiology of suicide. In: Jacobs DG (ed) The Harvard Medical School guide to suicide assessment and intervention. San Francisco, Jossey-Bass, 1999; 40-51. 